\title{
Investigating perceptually based models to predict importance of facial blendshapes
}

\author{
Emma Carrigan \\ Trinity College Dublin \\ Rozenn Dahyot \\ Trinity College Dublin
}

\author{
Katja Zibrek \\ INRIA, Rennes \\ Rachel McDonnell
Trinity College Dublin
ramcdonn@tcd.ie
}

\begin{abstract}
Blendshape facial rigs are used extensively in the industry for facial animation of virtual humans. However, storing and manipulating large numbers of facial meshes is costly in terms of memory and computation for gaming applications, yet the relative perceptual importance of blendshapes has not yet been investigated. Research in Psychology and Neuroscience has shown that our brains process faces differently than other objects, so we postulate that the perception of facial expressions will be feature-dependent rather than based purely on the amount of movement required to make the expression. In this paper, we explore the noticeability of blendshapes under different activation levels, and present new perceptually based models to predict perceptual importance of blendshapes. The models predict visibility based on commonly-used geometry and image-based metrics.
\end{abstract}

\section{CCS CONCEPTS}

- Applied computing $\rightarrow$ Psychology; • Computing methodologies $\rightarrow$ Mesh geometry models; $\bullet$ Mathematics of computing $\rightarrow$ Equational models.

\section{KEYWORDS}

action units, perception, linear model, blendshapes

\section{ACM Reference Format:}

Emma Carrigan, Katja Zibrek, Rozenn Dahyot, and Rachel McDonnell. 2020. Investigating perceptually based models to predict importance of facial blendshapes. In Motion, Interaction and Games (MIG '20), October 16-18, 2020, Virtual Event, SC, USA. ACM, New York, NY, USA, 6 pages. https: //doi.org/10.1145/3424636.3426904

\section{INTRODUCTION}

Virtual human expressions are generally created by animating blendshape rigs [Lewis et al. 2014] based on the Facial Action Coding System (FACS) [Ekman and Friesen 1978b]. However, these rigs are computationally expensive for real-time applications due to the large amounts of geometry processing. The question of importance of blendshapes is therefore of great interest to computer

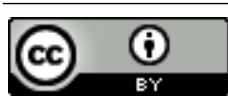

This work is licensed under a Creative Commons Attribution International 4.0 License.

MIG '20, October 16-18, 2020, Virtual Event, SC, USA

(c) 2020 Copyright held by the owner/author(s)

ACM ISBN 978-1-4503-8171-0/20/10.

https://doi.org/10.1145/3424636.3426904 games and other real-time applications, with the aim of reducing the number of blendshapes needed for animating a rig [Costigan et al. 2016], or prioritising which blendshapes to include in expressions for example-based rig creation algorithms [Carrigan et al. 2020]. Additionally, algorithms that create or alter facial geometry are usually evaluated against ground-truth facial meshes using standard geometry error metrics, however, we postulate that standard error-metrics may not be sufficient to determine how perceptually different the results are to the ground-truth.

Due to the fact that humans are particularly attuned to face perception [Bruce and Young 2013], we expect that differences in perception of facial action units will not align with the magnitude of displacement on the mesh caused by the expression. We hypothesise that small displacements in salient regions (e.g., eyelids) will be more perceptually noticeable than larger displacements in less salient regions (e.g., puffing of cheeks), which may not be accurately reflected by standard geometric and image error metrics. We also expect that the sex and race of the face will affect the perception of action units on that face, due to social conditioning [Hess et al. 2004; Lindsay et al. 1991].

In this paper, we investigate the perceptual impact of a carefully selected range of expressive action units at varying activation levels across a number of characters of different race and sex. We then compare our qualitative perceptual results to quantitative metrics in order to determine whether the perceptual effect can be predicted directly. We aim to determine if our question of perceived action unit importance can be predicted by calculating the error between the neutral pose and the expression blendshape, using both standard and perceptually based metrics, calculated from either the 3D geometry or the rendered 2D image. We then perform linear regression analysis to determine the best model describing the relationship between perception and error metrics. We address a number of questions, such as:

- Are certain facial action units more perceptually noticeable than others?

- Can we predict the saliency of facial action units using numerical error metrics, and is there a benefit to using existing perceptually based metrics?

- Are 3D geometry or 2D image-based metrics better at predicting the saliency of facial action units?

\section{RELATED WORK}

Face perception is a very active area of study in Psychology, as humans have been shown to have specialised sensory and interpretative processes related to faces [Bruce and Young 2013; Farah et al. 
1998; Kanwisher et al. 1997]. Work by Schwaninger et al. [2009; 2006] shows that faces are processed both in terms of their components, as well as the configuration of those components, rather than purely holistically. A great deal of research is ongoing in the areas of face recognition, detection, memory, the other-race effect and the effect of experience on face perception, critical features for recognition, and social evaluation of faces [Oruc et al. 2019].

Since particular areas of the face are important for the recognition of emotion [Smith et al. 2005], different action units could potentially be more salient than others. The evidence supporting this suggests that specialised areas exist in the brain (region pSTS) for the perception of action units. This could indicate that action units are a necessary precursor to categorization of emotion [Srinivasan et al. 2016]. In addition, particular action units are responsible for the correct recognition of an emotion [Wegrzyn et al. 2017].

There were also studies which used the information about individual action units to generate synthetic expressions. A gradual activation of specific action units resulted in detection of an expression [Yu et al. 2012]. Reverse engineering expressions based on perceptual relevance helped with improved facial recognition in artificial faces [Chen et al. 2018]. There is enough evidence to suggest that action units alone have a perceptually significant impact on emotion categorisation. However, it is unknown if certain action units are more salient than others because they are associated with a particular emotional expression.

While the mouth is understandably a significantly attended to area due to its importance for emotional expression and communication [Nusseck et al. 2008] and its size relative to other facial features, the eyes and eyebrows can also be considered highly important despite their considerably smaller size. Eyebrows are integral for emotional and conversational signals [Ekman 1979], and can alter the perception of the eyes [Matsushita et al. 2015], however they are important in their own right for face recognition [Sadr et al. 2003] and not just in relation to how they change the perception of eyes.

While there has been much research in the area of Psychology on perception of the human face, these results are rarely utilized in Computer Graphics to improve the quality or computation of facial animation for real-time applications where resources are limited. The current state of the art for high quality real-time facial animation is blendshape animation [Lewis et al. 2014]. There is currently no consensus on what blendshapes a rig should contain, with the decision being left entirely to the artist. One solution is to use the Action Units from the Facial Action Coding System [Ekman and Friesen 1978a]. In theory, FACS breaks down facial expressions to their most basic components, making it a useful guideline for blendshape creation.

Optimisation of blendshape animation can be done in a few ways. Reducing mesh complexity is one method [Garland and Heckbert 1997], however this causes correspondence issues between shapes. The animation itself can be optimised by passing blendshapes [Lorach 2007] and animation to the GPU, and using GPGPU methods [Costigan et al. 2016]. The most relevant optimisation method for this paper would be blendshape reduction, either removing blendshapes from a rig or from an animation. Naturally, this would reduce the expressivity of a rig and reduce the quality of animations, so identifying salient blendshapes as we attempt to do in this work is important.

Mesh optimisations in graphics have traditionally been assessed using error metrics, which are used to measure dissimilarity between ground-truth geometry and geometry after undergoing simplification, watermarking, or lossy compression, with the goal of avoiding perceptible differences. The types of metrics used are viewdependent and view-independent, or image-based and geometrybased (see overview by Corsini et al. [2013]). We are interested if these metrics can be used in face-geometry perception. In the context of facial animation, Deng et al. [2008] used a similar approach to ours by bridging objective facial motion patterns with subjective perceptual outcomes to predict the emotional fidelity of expressive facial animations.

\section{STIMULI CREATION}

We first acquired a high-end photogrammetry-scanned template model, created by Eisko ${ }^{1}$, a leading Digital Double company. The character had over 200 blendshapes, inspired by the FACS [Ekman and Friesen 1978b] with additional shapes for emotion and speech. Our experiment characters were a set of 6 high-resolution scanned neutral faces from 3D Scan Store ${ }^{2}$, including 2 characters of each Asian, Black, and White race. Within each race group, there was 1 female and 1 male character.

\subsection{Blendshape Transfer}

In order to obtain a range of expressions for each of our experiment characters, we used the Russian 3D Scanner ${ }^{3}$ Wrap 3.4 to transfer the topology of our template model to each of the neutral characters, using some feature points as guidance so that the semantics of the topology remained the same. We then used this wrapped mesh to warp the blendshapes of our template model to the experiment characters, thereby creating 6 new character rigs with equal topology and blendshapes. We chose not to include any hair on the characters as we are exclusively interested in facial features and wanted to avoid distracting elements.

\subsection{Action Unit Selection}

We carefully chose 11 blendshapes from the character's set of 200 for the experiment (see Figure 1). The AUs chosen are those which are important for emotion (AUs 2, 4, 5, 12, 15, 26, 38) [Wegrzyn et al. 2017], speech (AUs 18, 26) [Meng et al. 2018], and those necessary for realistic and natural motion (AU 43) [Itti et al. 2003]. The cheeks have also been found to be important for facial recognition [Busso et al. 2004], so in order to fully cover potentially important features we also included cheek AUs 34 and 35. We also attempted to include opposite movements in each area, e.g., smile and frown.

\subsection{Activation Levels}

We are interested in whether the increase in onset of an AU linearly affects its perceptual importance, or whether there is a point at which the AU becomes more noticeable. For this reason, we investigate each AU at a number of different levels of activation. For

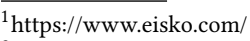

${ }^{2}$ https://www.3dscanstore.com/3d-head-models/

${ }^{3}$ https://www.russian3dscanner.com/
} 


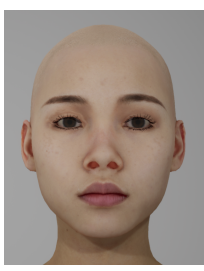

(a) Neutral

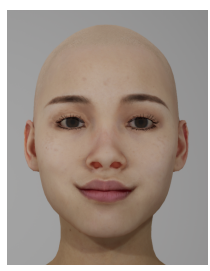

(e) Smile

Lips Closed

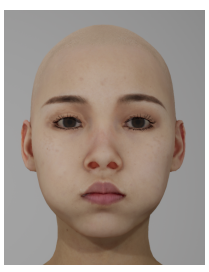

(i) Cheeks

Puffed

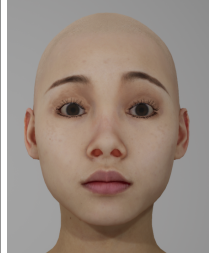

(b) Eyebrows Up

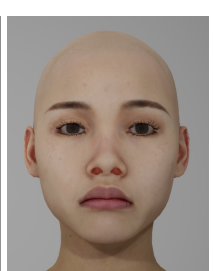

(f) Mouth Frown

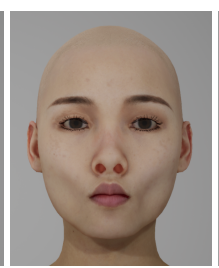

(j) Cheek

Inhaled

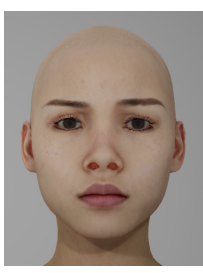

(c) Frown

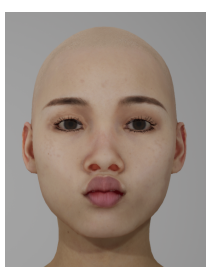

(g) Lips

Protrude

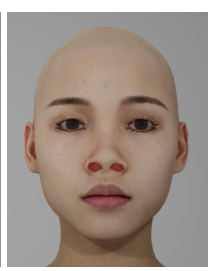

(k) Nostrils Dilated

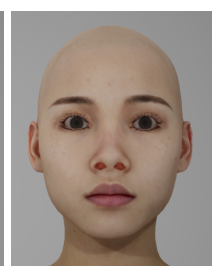

(d) Eyes Opened

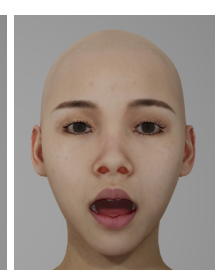

(h) Mouth Open

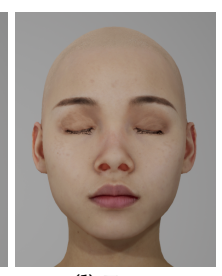

(l) Eyes

Closed
Figure 1: The blendshape set used in our experiment, shown on the Asian Female character at full activation (1.0).

each of these expressions, we show 5 activation levels: $0.2,0.4,0.6$, $0.8,1.0$, with 1.0 being the maximum activation of that expression performed by the actor during the scanning process. In terms of blendshapes, this is simply a linear interpolation from the neutral face to the blendshape, with 1.0 being the fully activated expression (e.g., eyes fully closed) and each intermediate step being a transition from neutral to that expression.

\section{PERCEPTUAL EXPERIMENT}

We chose to develop a real-time experiment system in Unreal Engine 4 for flexibility and so that we could utilize pre-built advanced lighting and shading for realistic virtual character visualisation. For each trial of the experiment, we displayed the Neutral expression on the left and the stimulus on the right, and asked the participants to answer "How different are the expressions?" using a slider. The slider ranged from 1 defined as "No Difference" to 9 defined as "Extremely Different". Participants were aware that the left image was always neutral. After each trial, a 1 second focus cross was displayed. We chose the Likert scale instead of a two-alternative forced-choice paradigm, in order to determine the relative saliency of AUs and activation levels, rather than simply whether the activation levels were noticed or not. Participants were asked to answer as quickly and accurately as possible.

At the beginning of the experiment, participants conducted a training session, where they completed 11 trials showing the full activated blendshapes on the template character, which was not used in the main experiment in order to calibrate participants to the most extreme examples of each AU. Three hundred and sixty trials were shown to participants in random order, 12 AUs $x 5$ activation levels $\times 6$ characters.

\subsection{Participants}

Twenty participants volunteered for the experiment ( 3 female, 16 male, 1 prefer not to answer; 8 were in the age range $18-27,10$ in 28-37, and 2 in 38-47). All reported medium or high familiarity with computer graphics and video games. As the experiment characters varied in race, and there is a perceptual effect of one's own race and perception of other races [Lindsay et al. 1991], we asked the participants to disclose their race (5 Asian, 13 White, 0 Black, 2 Other).

\subsection{Results}

We ran a 4-way repeated measures ANOVA on the perceptual difference results with the within factors Sex, Race, Action Unit, and Activation Level. Due to the imbalance between participant race and sex groups, we did not include these between-groups factors in the analysis. The ANOVA results can be seen in Table 1. We ran post-hoc analysis using Tukey's HSD tests throughout.

Table 1: ANOVA interactions with dependent variable "Difference" from the perceptual results. $(A U=$ Action Unit, * represents significant $\mathbf{p}$-values, $\mathrm{F}^{*}$ stand for GreenhouseGeisser correction for violations of sphericity). Effects sizes are reported in the last column $\left(\eta_{p}^{2}\right)$.

\begin{tabular}{l|r|r|r}
\hline Factor & $\mathbf{F}(\mathbf{D F n}, \mathbf{D F d})=\mathbf{F}$-value & p-value & $\eta_{p}^{2}$ \\
\hline Sex & $\mathrm{F}(1,19)=1.727$ & 0.2 & 0.08 \\
\hline Race & $\mathrm{F}(2,38)=4.192$ & $0.02^{*}$ & 0.18 \\
\hline Action Unit & $\mathrm{F}^{*}(2.93,55.58)=123.8$ & $0.00^{*}$ & 0.86 \\
\hline Activation & $\mathrm{F}^{*}(1.21,22.90)=158.2$ & $0.00^{*}$ & 0.89 \\
\hline Sex-Race & $\mathrm{F}(2,38)=7.826$ & $0.001^{*}$ & 0.29 \\
\hline Sex-AU & $\mathrm{F}(11,209)=2.99$ & $0.001^{*}$ & 0.14 \\
\hline Race-AU & $\mathrm{F}(22,418)=6.885$ & $0.00^{*}$ & 0.27 \\
\hline Sex-Activation & $\mathrm{F}(4,76)=2.887$ & $0.03^{*}$ & 0.13 \\
\hline Race-Activation & $\mathrm{F}(8,152)=1.581$ & 0.14 & 0.08 \\
\hline AU-Activation & $\mathrm{F}(44,836)=19.29$ & $0.00^{*}$ & 0.50 \\
\hline Sex-Race-AU & $\mathrm{F}^{*}(6.73,127.86)=5.301$ & $0.00^{*}$ & 0.22 \\
\hline Sex-Race-Activation & $\mathrm{F}(8,152)=2.031$ & $0.046^{*}$ & 0.10 \\
\hline Sex-AU-Activation & $\mathrm{F}(44,836)=0.979$ & 0.5 & 0.05 \\
\hline Race-AU-Activation & $\mathrm{F}(88,1672)=1.592$ & $0.001^{*}$ & 0.07 \\
\hline Sex-Race-AU-Activation & $\mathrm{F}(88,1672)=1.68$ & $0.00^{*}$ & 0.08 \\
\hline
\end{tabular}

4.2.1 Character Sex \& Race. There was no main effect of the character Sex. However, we found a main effect of character Race, where shape differences were less perceptible for Black characters overall than for Asian characters $(p<0.02)$. An interaction between Race and Sex gave further insight that shape differences were more perceptible for the Asian Female character than other characters except for the White Male ( $p<0.03$ for all). There was an interaction between Race and AU, which showed the Frown and Cheeks Puffed $(p<0.02)$ were the main AUs that were less perceptible on 
Table 2: The AUs ordered by average perceptual difference.

\begin{tabular}{|c|c||c|c|}
\hline AU Name & Difference & AU Name & Difference \\
\hline Mouth Open & 5.97 & Eyes Opened & 3.15 \\
Eyes Closed & 5.2 & Cheeks Puffed & 2.77 \\
Smile Lips Closed & 4.18 & Mouth Frown & 2.56 \\
Eyebrows Up & 3.56 & Frown & 2.22 \\
Lips Protude & 3.55 & Nostrils Dilated & 1.78 \\
Cheek Inhaled & 3.24 & Neutral & 1.42 \\
\hline
\end{tabular}

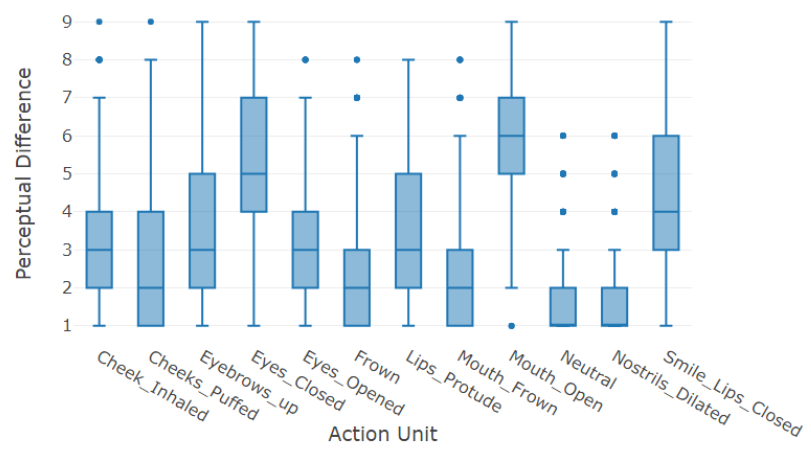

Figure 2: Main effect of AU from our experiment.

Black characters. These small differences across Race could have been due to our predominantly European and Asian participant pool [Lindsay et al. 1991; Walker and Tanaka 2003] or possibly due to differences in perception due to stand-out or distracting features in the rendering of their faces.

4.2.2 Activation Level. A main effect of Activation Level showed a significant increase in perceived differences as the activation increased, as expected. There was no difference across all characters and AUs at the lowest Activation Level of 0.2. However, some characters were rated as relatively more different at higher Activation Levels. Specifically, Asian Female at 0.6 Activation Level was rated similarly to the AUs of some characters at the 0.8 level.

4.2.3 Action Units. Mouth Open, Eyes Closed, and Smile Lips Closed appeared to have a higher perceptual effect since the perceived differences were significantly higher when compared to all other AUs $(p<0.02)$. Nostrils Dilated had the smallest effect since it was not significantly different from the Neutral. See Figure 2 and Table 2 .

Further interactions showed that Mouth Open was significantly more different than most other shapes $(p<0.005)$. Eyes Closed were also prominent on some characters, while Nostrils Dilated and Frown were not different from Neutral, for some characters.

Mouth Frown was the only AU to be rated significantly differently between the sexes $(p<0.05)$, with the female characters being rated as more different. This could potentially be related to the inverse effect of gender stereotyping increasing saliency of unexpected emotions seen in previous work (i.e., that females are perceived as more angry than males) [Hess et al. 2004]. We also found interactions with Race, as well as interactions with Race and
Sex (see Table 1). While we observed many significant differences from post-hoc tests, we did not observe any meaningful patterns.

\section{ERROR METRICS}

We now move to investigating the relationship between numerical error metrics and perception. We first record the error metric scores for each activation level of each $\mathrm{AU}$, for each character. Each metric is calculated between the neutral face and the activated AU.

\subsection{Geometric Error Metrics}

Root-Mean-Square. We calculate the RMS error between two meshes by getting the sum across all vertices $n \in N$ of the square root of the average of the square of each $(x, y, z)$ component of each delta vertex $\delta \vec{v}_{n}$, i.e. the difference between that vertex position in the blendshape mesh and the same vertex in the neutral mesh, as described in Equation 1.

$$
\delta_{R M S}=\sum_{n=1}^{N} \sqrt{\frac{1}{3} \delta \vec{v}_{n}^{T} \delta \vec{v}_{n}}=\frac{1}{\sqrt{3}} \sum_{n=1}^{N}\left\|\delta \vec{v}_{n}\right\|
$$

Spatio-Temporal Edge Difference. STED is a perceptual metric for dynamic meshes which focuses on local and relative changes of edge length by measuring the standard deviation of relative edge length around each vertex, rather than global mesh difference. The model parameters have been tuned such that its results best match those of the perceptual experiment, described in the paper [Vasa and Skala 2010].

\subsection{Image Error Metrics}

To calculate our image metric results, we took screenshots of each stimulus and cropped out a large amount of the empty space surrounding each head. MSE and SSIM were calculated using scikitimage [van der Walt et al. 2014].

Mean-Squared-Error. We calculate MSE by getting the per-pixel average error between images $A$ and $B$, where $N$ is the total number of pixels in the image, and $\vec{x}_{n}^{A}$ is the $n^{t h}$ pixel of image A.

$$
\delta_{M S E}=\frac{1}{N} \sum_{n=1}^{N} \vec{x}_{n}^{A}-\vec{x}_{n}^{B}
$$

Structural Similarity Index Metric. SSIM is calculated as defined by Wang et al. [2004] and using the default suggested parameters. It is designed to model the response of the human vision system and should correlate better to our perceptual results than standard MSE. As this metric measures similarity rather than difference, we invert this metric (1-SSIM) for better comparison with our other metrics where appropriate.

\section{MODEL FIT}

To find the best model describing the relationship between perceptual results and the calculated errors, several Generalised Linear Models were tested and compared using Akaike Information Criterion (AIC) that combines the log-likehood (best fit) penalised by the model complexity (as measured by the number of parameters to estimate in the model) for selection of the best model [Dobson and Barnett 2008]. The model with the lowest AIC is deemed the best 
model (amongst those tested) for explaining the observations. A $\chi^{2}$ test for the deviance is then used to assess if this selected 'best' model is actually a good model for explaining the data [Dobson and Barnett 2008]. Poisson and Gaussian distributions were tested in combination with several link functions (identity, log, and square root). We found that the Poisson distribution captures the discrete nature of the perceived difference best and provides lower AICs than with the Gaussian distribution in the many models tested including the ones shown in Table 3.

Table 3: Model comparison with AIC $\downarrow$ to explain the perceived difference (columns 2 and 3). Best link function reported between Identity (Id), log and sqrt. The lowest AIC for each metric are displayed in bold. Deviances (all with Poisson distribution and best link function) for models are shown column 4. A good model has a deviance in the interval $\left[0 ; \chi_{0.95}^{2}\right]$ with $\chi_{0.95}^{2}$ reported in column 5 .

\begin{tabular}{|c|c|c|c|c|}
\hline Model & Gaussian & Poisson & Deviance & $\chi_{.95}^{2}$ \\
\hline STED & 28704 (Id) & 27346 (Id) & 6551 & 7396 \\
\hline $\mathrm{STED}^{*} \mathrm{AU}$ & 24850 (sqrt) & 24680 (sqrt) & 3844 & 7374 \\
\hline STED*AU+Race:Sex & 24832 (sqrt) & 24670 (sqrt) & 3823 & 7368 \\
\hline STED $^{*} \mathrm{AU}^{*}$ Sex ${ }^{*}$ Race & 24781 (sqrt) & 24742 (sqrt) & 3676 & 7252 \\
\hline RMS & 27965 (Id) & 26903 (Id) & 6109 & 7396 \\
\hline $\mathrm{RMS}^{*} \mathrm{AU}$ & 24878 (Id) & 24688 (Id) & 3852 & 7374 \\
\hline RMS*AU+Race:Sex & 24853 (Id) & 24673 (Id) & 3827 & 7368 \\
\hline $\mathrm{RMS}^{*} \mathrm{AU}^{*}{ }^{*}$ ex ${ }^{*}$ Race & 24810 (Id) & 24749 (Id) & 3683 & 7252 \\
\hline SSIM & 29534 (Id) & 28075 (Id) & 7280 & 7396 \\
\hline $\operatorname{SSIM}^{*} \mathrm{AU}$ & 26287 (Id) & 25591 (Id) & 4753 & 7374 \\
\hline SSIM*AU+Race:Sex & 25505 (sqrt) & $25112(\log )$ & 4264 & 7368 \\
\hline SSIM $^{*} \mathrm{AU}^{*}{ }^{*} \mathrm{Sex}^{*}$ Race & 24799 (sqrt) & $24758(\log )$ & 3680 & 7252 \\
\hline MSE & 29920 (Id) & 28678 (Id) & 7884 & 7396 \\
\hline $\mathrm{MSE}^{*} \mathrm{AU}$ & $26940(\log )$ & $26120(\log )$ & 5277 & 7374 \\
\hline MSE*AU+Race:Sex & 25933 (Id) & 25384 (Id) & 4536 & 7368 \\
\hline $\mathrm{MSE}^{*} \mathrm{AU}^{*} \mathrm{Sex}^{*}$ Race & 24839 (Id) & 24776 (Id) & 3698 & 7252 \\
\hline
\end{tabular}

\subsection{Variable selection with ANOVA}

The variables used to design the models shown in Table 3 have been chosen using ANOVA. Table 4 in the Supplemental Material shows the ANOVA when fitting a linear regression to explain the Perceived Difference (response variable) with explanatory variables RMS, AU and the 6 experiment characters (captured with variables Sex and Race). As can be seen by the high values for Sum Sq., most of the perceived difference is explained using RMS and AU with their interactions (variable highlighted in green in Table 4). Similarly, Table 5 in the Supplemental Material shows the ANOVA with explanatory variables STED, AU and the 6 virtual characters used and the high values for Sum Sq. imply that most of the perceived difference is explained using STED and AU with their interactions (variable highlighted in green Table 5). These results imply the relationship between the perceived values and the geometry metrics are AU-specific, and using an AU-specific model is necessary for prediction. ANOVA tables for image metrics are shown likewise in Table 6 and 7 in the Supplemental Material. We note that MSE and SSIM alone have less explanatory power than RMS and STED variables (see lower Sum Sq. in the tables). These ANOVA tables explain the comparison shown in Table 3 where AICs of models shown are either using only the metrics (Metric=STED/RMS/SSIM/MSE), the full models (Metric ${ }^{*} \mathrm{AU}^{*}$ Sex ${ }^{*}$ Race), the ones considering interactions between metrics and blendshapes (Metric*AU), and the models that include Sex and Race as additional variables.

\subsection{Best Metric?}

In the geometry domain, all fitted models are good models as per their deviance reported in Table 3 [Dobson and Barnett 2008]. However, we note that the perceptual metric STED achieves a lower AIC (marginally) in comparison to the standard metric RMS (see Table 3). Similarly, in the image domain, the perceptual metric SSIM achieves a lower AIC (marginally) in comparison to the standard metric MSE (Table 3). All fitted models are good models as per their deviance reported in Table 3 with the exception of the simplest one using only MSE. This shows that MSE has less explanatory power than SSIM for explaining the perceived difference, which is not surprising since it does not account for structural fidelity of the image. Figure 3 in the Supplemental Material illustrates the different models used.

We found that the perceptual image metric SSIM (measured in a $2 \mathrm{D}$ projective space) is not as powerful as even the standard geometry metric RMS (measuring the deformation in 3D) for explaining the perceived difference.

This is interesting, as our participants viewed the stimuli as a $2 \mathrm{D}$ projection, however their recorded perceived difference is better explained by geometric metrics computed from 3D meshes. A potential explanation may be that because faces are very familiar objects, a 3D representation is automatically imagined or inferred by participants when viewing $2 \mathrm{D}$ facial images. Despite this, having a model fitted using image metrics can be useful for prediction of perceived difference when geometry metrics are not available (e.g., for facial photograph comparisons).

\section{DISCUSSION}

In this paper, we presented the first experiment on perceptibility of facial action units, and the relationship with numerical metrics describing the displacements. Our main contribution is our perceptual models, which will provide a starting point for the development of a universal perceptual error metric suitable for human faces ${ }^{4}$.

Additionally, we found that some facial action units were more perceptually noticeable than others, and provide a table showing the order of importance (Table 2). This perceptual ordering will be useful for game developers for tasks that require an order of blendshapes, such as level-of-detail blendshape reduction methods [Costigan et al. 2016], or example creation for blendshape transfer [Carrigan et al. 2020]. By identifying and removing blendshapes of lower visual saliency, which equates to simply removing rows from the blendshape matrix, we can save both memory and computation required.

In general, we found an equally-spaced linear relationship between perceptual difference and activation level. This implies that future experiments can focus solely on the highest activation level (1.0) of an AU.

\footnotetext{
${ }^{4}$ Data and R code used for this study are shared at https://github.com/Roznn/facialblendshapes allowing others to build on our data investigating a larger range of faces, viewpoints, and facial action units.
} 
In terms of the error metrics, one of our aims was to determine if existing standard and perceptual geometry- and image-based error metrics could predict the perceptibility of facial action units when compared to the neutral face. We did not find this to be the case, as our statistical models showed that each AU had a different relationship with the metrics (see different slopes in Supplemental Material, Figure 3). It is interesting how no single geometric or image-based metric alone was able to capture well the perceptual difference as seen by a human, even by the metrics which are based on the human vision system. This implies an AU-specific perception that must be taken into account when measuring the perceptual impact of changes in facial expressions, which is not catered for by the current metrics used alone in the models.

Interestingly, we found that image metrics were worse at predicting perceived differences than geometry metrics, even though the viewers only viewed the 3D geometry from a single viewpoint. This implies that humans have a strong ability to infer 3D shape of faces from a 2D image, and that the pixel-based differences in the images do not capture these differences as well as 3D geometry comparisons. This is unlikely to hold true for different viewpoints besides the front view, but will be interesting to investigate in future work.

In this work, we focused on realistic virtual human faces, so it is not clear if our results would generalize to other character rigs or even real faces. It would be interesting to investigate stylized or cartoon character rigs in future work. Also, as this is an initial study into investigating the perceived importance of facial AUs, we limited our study to static expressions of single AUs. Naturally, perception of animated faces with combined expressions would be more complicated, particularly since specific AUs are important for the perception of emotions (e.g., AU 7 Lid Tightener for anger [Wegrzyn et al. 2017]). It might be the case that even if these AUs are not perceptually important according to our approach, removing them from a rig might alter the interpretation of emotion of a virtual human, which we will study in future work.

\section{ACKNOWLEDGMENTS}

This work was funded by Science Foundation Ireland under the ADAPT Centre for Digital Content Technology (Grant 13/RC/2106) and the Game Face project (Grant 13/CDA/2135).

\section{REFERENCES}

Vicki Bruce and Andy Young. 2013. Face perception. Psychology Press.

Carlos Busso, Zhigang Deng, Serdar Yildirim, Murtaza Bulut, Chul Min Lee, Abe Kazemzadeh, Sungbok Lee, Ulrich Neumann, and Shrikanth Narayanan. 2004. Analysis of emotion recognition using facial expressions, speech and multimodal information. In Proceedings of the 6th international conference on Multimodal interfaces. 205-211.

Emma Carrigan, Eduard Zell, and Rachel McDonnell. 2020. Expression Packing: AsFew-As-Possible Training Expressions for Blendshape Transfer. In Proceedings of the 41st Annual European Association for Computer Graphics Conference, Vol. 39. Eurographics Association, 219-233.

Chaona Chen, Oliver GB Garrod, Jiayu Zhan, Jonas Beskow, Philippe G Schyns, and Rachael E Jack. 2018. Reverse Engineering Psychologically Valid Facial Expressions of Emotion into Social Robots. In Int. Conf. on Automatic Face \& Gesture Recognition 448-452.

Massimiliano Corsini, Mohamed-Chaker Larabi, Guillaume Lavoué, Oldřich Petřík, Libor Váša, and Kai Wang. 2013. Perceptual metrics for static and dynamic triangle meshes. In Computer Graphics Forum, Vol. 32. Wiley Online Library, 101-125.

Timothy Costigan, Anton Gerdelan, Emma Carrigan, and Rachel McDonnell. 2016. Improving blendshape performance for crowds with GPU and GPGPU techniques. In Proceedings of the 9th International Conference on Motion in Games. ACM, 73-78.
Zhigang Deng and Xiaohan Ma. 2008. Perceptually Guided Expressive Facial Animation. In Proceedings of the 2008 ACM SIGGRAPH/Eurographics Symposium on Computer Animation (Dublin, Ireland) (SCA '08). Eurographics Association, Goslar, DEU, 67-76.

A. J. Dobson and A. G. Barnett. 2008. An Introduction to Generalized Linear Models. CRC Press, Third Edition.

Paul Ekman. 1979. About brows: Emotional and conversational signals. Human Ethology (1979).

Paul Ekman and Wallace V Friesen. 1978a. Facial action coding system. Consulting Psychologists Press, Stanford University.

Paul Ekman and Wallace V. Friesen. 1978b. Facial Action Coding System: A Technique for the Measurement of Facial Movement. Consulting Psychologists Press.

Martha J Farah, Kevin D Wilson, Maxwell Drain, and James N Tanaka. 1998. What is "special" about face perception? Psychological review 105, 3 (1998), 482.

Michael Garland and Paul S Heckbert. 1997. Surface simplification using quadric error metrics. In Proceedings of the 24th annual conference on Computer graphics and interactive techniques. 209-216.

Ursula Hess, Reginald B Adams Jr, and Robert E Kleck. 2004. Facial appearance, gender, and emotion expression. Emotion 4, 4 (2004), 378.

Laurent Itti, Nitin Dhavale, and Frederic Pighin. 2003. Realistic avatar eye and head animation using a neurobiological model of visual attention. In Applications and Science of Neural Networks, Fuzzy Systems, and Evolutionary Computation VI, Vol. 5200. International Society for Optics and Photonics, 64-78.

Nancy Kanwisher, Josh McDermott, and Marvin M Chun. 1997. The fusiform face area: a module in human extrastriate cortex specialized for face perception. Fournal of neuroscience 17, 11 (1997), 4302-4311.

John P Lewis, Ken Anjyo, Taehyun Rhee, Mengjie Zhang, Frederic H Pighin, and Zhigang Deng. 2014. Practice and Theory of Blendshape Facial Models. Eurographics (State of the Art Reports) 1, 8 (2014), 2.

D Stephen Lindsay, Philip C Jack, and Marcus A Christian. 1991. Other-race face perception. Journal of applied psychology 76, 4 (1991), 587.

Tristan Lorach. 2007. Directx 10 blend shapes: Breaking the limits. GPU Gems 3 (2007), 53-67.

Soyogu Matsushita, Kazunori Morikawa, Saya Mitsuzane, and Haruna Yamanami. 2015. Eye shape illusions induced by eyebrow positions. Perception 44, 5 (2015), 529-540.

Zibo Meng, Shizhong Han, Ping Liu, and Yan Tong. 2018. Improving speech related facial action unit recognition by audiovisual information fusion. IEEE transactions on cybernetics 49,9 (2018), 3293-3306.

Manfred Nusseck, Douglas W Cunningham, Christian Wallraven, and Heinrich H Bülthoff. 2008. The contribution of different facial regions to the recognition of conversational expressions. Journal of vision 8, 8 (2008), 1-1.

Ipek Oruc, Benjamin Balas, and Michael S Landy. 2019. Face perception: A brief journey through recent discoveries and current directions. Vision Research 157 (2019), 1-9.

Javid Sadr, Izzat Jarudi, and Pawan Sinha. 2003. The role of eyebrows in face recognition. Perception 32, 3 (2003), 285-293.

Adrian Schwaninger, Janek S Lobmaier, Christian Wallraven, and Stephan Collishaw. 2009. Two routes to face perception: evidence from psychophysics and computational modeling. Cognitive science 33, 8 (2009), 1413-1440.

Adrian Schwaninger, Christian Wallraven, Douglas W Cunningham, and Sarah D Chiller-Glaus. 2006. Processing of facial identity and expression: a psychophysical, physiological, and computational perspective. Progress in Brain Research 156 (2006), 321-343.

Marie L Smith, Garrison W Cottrell, FrédéAric Gosselin, and Philippe G Schyns. 2005. Transmitting and decoding facial expressions. Psychological science 16, 3 (2005), 184-189.

Ramprakash Srinivasan, Julie D Golomb, and Aleix M Martinez. 2016. A neural basis of facial action recognition in humans. Fournal of Neuroscience 36, 16 (2016), 44344442.

Stéfan van der Walt, Johannes L. Schönberger, Juan Nunez-Iglesias, François Boulogne, Joshua D. Warner, Neil Yager, Emmanuelle Gouillart, Tony Yu, and the scikit-image contributors. 2014. scikit-image: image processing in Python. Peerf 2 (2014), e453.

Libor Vasa and Vaclav Skala. 2010. A perception correlated comparison method for dynamic meshes. IEEE transactions on visualization and computer graphics 17, 2 (2010), 220-230.

Pamela M Walker and James W Tanaka. 2003. An encoding advantage for own-race versus other-race faces. Perception 32, 9 (2003), 1117-1125.

Zhou Wang, Alan C Bovik, Hamid R Sheikh, and Eero P Simoncelli. 2004. Image quality assessment: from error visibility to structural similarity. IEEE transactions on image processing 13, 4 (2004), 600-612.

Martin Wegrzyn, Maria Vogt, Berna Kireclioglu, Julia Schneider, and Johanna Kissler. 2017. Mapping the emotional face. How individual face parts contribute to successful emotion recognition. PloS one 12, 5 (2017).

Hui Yu, Oliver GB Garrod, and Philippe G Schyns. 2012. Perception-driven facial expression synthesis. Computers \& Graphics 36, 3 (2012), 152-162. 